\title{
Tensile and creep behaviour of polyethylene terephthalate and polyethylene naphthalate fibres
}

\author{
C. Lechat ${ }^{1}$, A. R. Bunsell ${ }^{2,}{ }^{*}$, P. Davies ${ }^{3}$ \\ ${ }^{1}$ ERA Technology, Cleeve Road, Leatherhead, KT22 7SA, United Kingdom. \\ ${ }^{2}$ Centre des Matériaux - Mines ParisTech, CNRS UMR 7633, BP 87, 91003 Evry Cedex, France. \\ ${ }^{3}$ Materials \& Structures group, IFREMER, BP 70, 29280 Plouzané, France. \\ *: Corresponding author: A. R. Bunsell, Tel. +33160763015 Fax +331607631 50, email address : \\ anthony.bunsell@mines-paristech.fr
}

\begin{abstract}
:
Ropes made of twisted polyester (PET) yarns have been replacing traditional steel ropes and chains as mooring lines for offshore platforms in deep-sea environments. In order to optimise rope manufacture and the design of mooring systems, a thorough understanding of the material's mechanical behaviour is necessary. Besides PET, other materials can also be considered such as PEN, as it also a polyester similar to PET but stiffer. This paper presents a study and comparison of PET and PEN fibres' mechanical behaviour, based on experiments carried out on single filaments. Both fibres show similar non-linear tensile behaviour, with an evolution of modulus in four steps. The same microstructural model is proposed for both fibres, based on microfibrils aligned along the fibre axis and composed of an alternation of amorphous, mesamorphous and crystalline phases. The shape of the tensile loading curve is explained by the successive loading of these phases. Creep behaviour is also evaluated by considering the evolution of creep rate with applied load. This evolution is again similar for PET and PEN, both from a qualitative and quantitative point of view. The similarity in creep rate values for both fibres indicates that the microstructural mechanisms involved in creep may differ from those involved in short term tension loading.
\end{abstract}




\section{Introduction}

Polyethylene Terephthalate (PET) fibres represent a high proportion of all the technical fibres produced in the world. Among the various applications of this fibre, one recently developed is the manufacture of high strength mooring ropes for floating offshore platforms. For deepwater applications, the usual mooring system consisting of steel chains and wire ropes becomes too heavy and therefore a lighter replacement material needs to be used. PET has been found to be one of the best candidates, as it presents a good compromise of all required properties (high modulus, low density, good resistance in sea water). PET ropes have been installed in oilfields for the past 10 years [1-3]. Polyethylene Naphthalate (PEN) is also a polyester fibre, developed more recently [4-6], and has currently few applications in ropes. It presents similar properties to PET with a higher modulus, which could make it an attractive competitor for offshore mooring lines.

In service, mooring lines are constantly under cyclic loading that is always greater than zero and are therefore subject to continuous elongation due to creep. Since the mooring ropes' function is to limit the platform offset, a thorough understanding of the creep behaviour of the material is necessary to optimise the design of such ropes. With this objective in mind, this paper presents an experimental study on the tensile and creep behaviour of PET and PEN, performed at the scale of single filaments. A second paper will examine the transfer of these properties to ropes.

\section{Materials}

The molecular structures of both polymers are shown on Figure 1. They are similar, but the naphthalene ring in PEN replacing the benzene ring in PET should confer a higher stiffness to the structure.

The fibres that have been tested in this study (fibre meaning here single filaments) were extracted from highly drawn PEN and PET multifilament yarns manufactured by Performance Fibers. PEN fibres were provided as 1100 dtex yarns, one yarn containing 140 fibres and each fibre having an average diameter of $27.9( \pm 1.5) \mu \mathrm{m}$. PET fibres were provided as 1100 dtex yarns, one yarn containing 192 fibres, each fibre having an average diameter of $22.9( \pm 0.9) \mu \mathrm{m}$. Both types of fibre were seen to be cylindrical using scanning electron microscopy (SEM).

For mechanical testing, single fibres were carefully extracted from the yarn manually and held at both ends in small cardboard tabs pasted with neoprene glue. The tabs were separated by a measured length of $50 \mathrm{~mm}$ representing the samples' gauge length

\section{Test procedure}

First, measurements of fibre diameter were made on each sample using a Mitutoyo LSM-6000 scanning laser apparatus. Tensile and creep tests were conducted in the fibre direction with an apparatus called the Universal Fibre Tester (see Figure 2) [7]. The device consists of two clamps, one directly fixed on the frame, and the other on a mobile cross-head. The cross-head displacement, thus the distance between clamps, was controlled by an electric motor and measured to within $1 \mu \mathrm{m}$ with a LVDT transducer. Constant load, constant displacement, or a computer controlled loading pattern could be imposed on the fibre. The load applied to the fibre was measured by a load cell $(0.01 \mathrm{~g}$ 
sensitivity). Load and displacement data were then collected using a standard software package (winATS), and analyzed in order to obtain stress and strain data.

For both tensile and creep tests, the load was applied with a strain rate of approximately $20 \%$ / minute. All tests were performed in a controlled environment, at $20^{\circ} \mathrm{C}$ and $50 \% \mathrm{RH}$.

For tensile data, 30 specimens were tested for both materials.

Creep tests were performed on both materials at loads ranging from 5 to $85 \%$ of their tensile strength. If samples had not failed after 24 hours, tests were stopped.

\section{Experimental data}

\section{Tensile tests}

Stress-strain curves for several specimens of PET and PEN fibres are shown, respectively in Figures $3 \mathrm{a}$ and $3 \mathrm{~b}$, and reveal the scatter in behaviour. The breaking load for PET varied between 0.4 and $0.5 \mathrm{~N}$, whereas for PEN it varied between 0.6 and $0.8 \mathrm{~N}$. Since PET and PEN fibres have different average diameters, it was necessary to normalise the load data to obtain comparable values. In the textile industry, normalisation is generally done using the linear mass of the material, in tex $(1$ tex $=1 \mathrm{~g} / \mathrm{km})$. The tex value of each tested filament was determined based on the density of the material (indicated by the manufacturer) and the filament's diameter (average value of three measurements along the length). The average strength measured on filaments for this study is $0.87 \mathrm{~N} /$ tex for PET and $0.92 \mathrm{~N} /$ tex for PEN, with respectively, standard deviations of 0.7 and $0.4 \mathrm{~N} /$ tex. Normalised curves are shown in Figure 4 . The breaking strain measured on PET fibres was in the range from 10 to $20 \%$, whereas for PEN it varied from 6 to $9 \%$. The initial modulus, defined as the slope of the stress-strain curve between zero and $1 \%$ strain, was evaluated as $10 \mathrm{~N} /$ tex for PET and $20 \mathrm{~N} /$ tex for PEN. The shape of tensile curves is typical of those found for PET and PEN fibres in the literature, revealing a non-linear behaviour.

\section{Creep tests}

Examples of strain versus time curves obtained from creep tests at various loads are given in Figure $5 a$ for PET and $5 b$ for PEN. The evolution of strain can be seen to have been linear, on a logarithmic time scale, for PET, as is generally described in literature. A similar behaviour is observed with PEN. For both materials, the creep rate is rather low (tenths of \% strain per decade) and appears to be dependant on the load applied. This dependence is not linear, as can be seen on Figure 6: for both fibres the creep rate is higher at $80 \%$ load than $40 \%$, but also higher at $20 \%$ than $40 \%$. Failure occurred within 24 hours only for the highest loads ( $\geq 80 \%$ of breaking strength).

\section{Data Analysis}

\section{Tensile behaviour}

The non-linearity of both fibres' tensile behaviour was considered by plotting the evolution of their modulus (instantaneous modulus, described as the slope of the stress-strain curve at each point) as a function of strain and also as a function of load. These plots are shown in Figures $6 \mathrm{a} \& 6 \mathrm{~b}$. and represent the moduli of PET 
and PEN filaments presenting average values of breaking strength and elongation at break (represented by a thick black curve in Figure $4 \mathrm{a}$ and $4 \mathrm{~b}$ ). The scale on the left hand side of each graph corresponds to PET, the one on the right hand side to PEN. In both cases, the modulus appears to vary showing four stages: a short initial increase to a peak value, then a rapid decrease to a minimum, followed by another progressive increase, and then finally the modulus decreases again until failure occurs.

For polyester fibres, the evolution of modulus during tensile test is generally described, in the literature, as occurring in three stages: a first decreasing slope associated with the motion of amorphous phases, a second stage during which the modulus increases as the molecular backbone phase is loaded, then a final stage of modulus decrease that could be due either to macromolecular chains sliding or failing [8]. These three steps would correspond to the last three observed on Figure 6, being preceded by the short initial increase which may have been overlooked, and could be considered as the initiation of the elongation: there is resistance to motion due to internal friction, which disappears as the motion begins.

This first increasing stage is reported elsewhere [9], in a study performed not on single filaments but on yarns. The author associates it with disentanglement of carbon chains. The graph presenting modulus evolution shows an initial modulus equal to zero, whereas in the present study the initial value was seen to be relatively high and close to its maximum value. This discrepancy is due to the tests in the present study being performed on single filaments, whereas the tests mentioned in the literature were performed on multi-filaments yarns. The zero initial value in the latter case may be due to scatter in filament lengths in the specimens: the filaments composing the yarn are not loaded simultaneously but successively, the shortest ones being loaded first and the longest ones last, leading to a progressive increase in modulus [10].

When comparing the evolution of modulus for PET and PEN fibres, similar behaviours are observed. Both polymers show the 4-stage evolution. Curves for PET and PEN modulus as a function of strain are nearly superimposed up to the second peak. Since the scale applicable for the curve of PEN modulus (righthand side scale) is exactly double of that applicable for the curve of PET modulus (left-hand side), the modulus of PEN can be estimated to be double of that of PET.

These similarities between PET and PEN indicate the possibility that their microstructures' morphologies are comparable and that identical mechanisms are involved when both fibres are subject to tensile forces.

The initial step, which is associated with internal friction phenomena, is very short and practically negligible: the initial modulus value is very close to the first peak value in comparison with the range between extremes. That possibly explains why previous studies do not usually refer to this initial increase.

The second step is related to the reorganisation of the amorphous phase. Considering the curves represented on Figure $6 \mathrm{a}$, it appears that this is limited to an elongation of $2 \%$. Once this strain limit is reached, the modulus starts to increase as the polymer backbone starts to take the load. Looking at Figure $6 \mathrm{~b}$, this increasing phase appears to be limited to a value of approximately $0.5 \mathrm{~N} / \mathrm{tex}$. Beyond this value the failure process begins.

The morphology of PET fibres has been studied by different authors and can be described as follows [11-12]: macromolecules are grouped in fibrils, in which can be observed an alternation of crystalline and amorphous zones. Fibrils are linked 
together by an interfibrillar amorphous phase that is slightly oriented along the fibre axis, called the mesamorphous phase (see Figure 7a). The microstructural model can be schematically represented as shown in Figure $7 \mathrm{~b}$.

When relating the microstructural model to the evolution of the microstructure during tensile loading, the 3-phase model (crystalline, amorphous and mesamorphous) seems coherent. However, the model proposed for the organisation of these three phases in the material is not completely satisfactory. The first decrease in modulus is thought to be due to the alignment of the fully amorphous phases. Looking at Figure $7 a$, it appears that the mesamorphous phase would be loaded and taut before the amorphous phase. The movement would be limited by this inter-fibrillar phase, which is already slightly aligned. That the mesamorphous phase should be the first one to be under load seems in contradiction with a decreasing modulus. A model of microstructure that would include some amorphous phase in the inter-fibrillar zone would seem more appropriate. Such a model has been suggested previously in a study on the fatigue mechanisms of PA66 fibres [13]. The mechanical behaviours of PET and PA66 fibres are similar, both fibres presenting similar changes of the stress-strain curve in tensile loading. Therefore, it seems justifiable to apply the microstructural model suggested for PA66 to PET and PEN fibres. An illustration of this model is given in Figure $8 \mathrm{a}$, and schematised in Figure $8 \mathrm{~b}$. We suggest improving this model by considering it as a parallel model as shown in Figure 8c, as experience has shown the existence of a distinct inter-fibrilar zone which is not represented in model $b$. The progressive transition along the fibre in each fibril from a fully crystalline phase to a mesamorphous phase and then an amorphous phase seems more realistic than going directly from a fully crystalline to a fully amorphous phase as in Figure 7. The model in Figure 8 differs from the classical model of Figure 7 in that the presence of a fully amorphous phase that can potentially be the first one to be loaded in the inter-fibrillar zone is now in agreement with the evolution of modulus.

If the numerical values of modulus for PET and PEN fibres are compared, there appears to be a contradiction with the microstructural model proposed above. Figure 6a shows that the modulus of PEN is, from the beginning of the tensile test, approximately twice that of PET. This is generally explained by the presence of the naphthalene ring on the PEN molecule, whereas PET only contains a single aromatic ring (see Figure 1). However our interpretation of the evolution of the modulus implies that the backbone of the molecule is not initially under load. The higher initial modulus of PEN may then be due, not to its stiffer backbone, but to the difference in size with the PET molecule: the steric crowding is more important for PEN because of the presence of the naphthalene ring, and this certainly plays a role in the tensile behaviour of the polymer.

\section{Creep behaviour}

For all creep curves shown in Figure 5 and for additional creep curves obtained at various loads, the creep rate is determined by fitting the curves with a logarithm function as follows: $\varepsilon(t)=A^{*} \log (t)+B$, where $\varepsilon$ is the strain value in $\%$, $t$ is time, $A$ and $B$ are constants. The coefficient $A$ is the creep rate, expressed in $\%$ strain per decade of time. When plotted as a function of the applied load, the evolution of the creep rate $A$ appears to be non-linear (see Figure 9). In contrast to that which might instinctively be expected, the creep rate does not continuously increase with the applied load. The evolution of the creep rate is very similar for both PET \& PEN fibres, and three stages can be distinguished. At very low loads, up to approximately $0.1 \mathrm{~N} / \mathrm{tex}$, it increases to a local maximum value of around $0.15 \%$ strain/decade of time. It then slightly decreases to a local minimum value of $0.1 \% /$ decade at a load of $0.35 \mathrm{~N} / \mathrm{tex}$, before increasing rapidly 
to finally reach high values leading to rapid failure of the specimens. Similar nonlinear viscoelastic creep behaviour has been observed on aramid fibres and the various phases of creep evolution have been related to the evolution of modulus during tension [14]. Some authors consider that similar microstructural mechanisms take place. However, our results seem to be in contradiction with such a hypothesis. In Figure 9, the ordinate axis, showing the strain rate, is the same for both materials. The similarity of behaviour for PET and PEN is quite remarkable: not only do they show similar evolution, but the numerical values are the same, whereas in tension the modulus of PEN is about twice that of PET. As previously mentioned, the difference in moduli can be explained by the presence of the double aromatic ring in the molecular structure of PEN whereas PET only contains a single ring. This seems to imply that the mechanisms taking place during creep neither involve the stretching of the backbone nor steric crowding. Creep could be related to another mechanism, involving the rupture of transverse bonds between macromolecules. These secondary bonds rely on Van der Waals forces. This hypothesis is confirmed by the observation that the nature of these secondary bonds is the same for PET and PEN. These bonds are also broken during tensile loading; however their failure remains a second order mechanism compared to the transformation of the molecular chains. During creep, it becomes the dominant mechanism taking place.

One question remains concerning the three stages in the creep rate evolution: the increasing rate with load during the initial and final phase is an evolution that could be expected. However, the decrease observed during the second phase is not yet explained.

\section{Conclusions}

The tensile behaviours of PET and PEN fibres were found to be very similar. Both fibres presented a four-stage evolution of modulus during tensile tests: an initial short increase related to the resistance to motion due to internal friction phenomena, a decrease, up to $2 \%$ strain, related to the alignment of amorphous chains, an increase due to the loading of the polymer backbone limited in force to a value of $0.5 \mathrm{~N} / \mathrm{tex}$, and a final decrease due to chains sliding or failing leading to the breakage of the fibre. The modulus of PEN was found to be twice that of PET up to approximately $3 \%$ strain. A similar microstructural model was suggested for both fibres.

The creep behaviours of PET and PEN were also shown to be very similar. When plotted as a function of applied load, the creep rate showed a three-step evolution: an initial short increase, a slight decrease, and then finally a sharp increase. The evolution of the creep rate of PEN fibres was qualitatively and quantitatively the same as that of PET. This led to the hypothesis that the phenomena taking place at a microstructural level during creep differ from those which occur in monotonic tensile loading, and do not involve the loading of the polymer backbone, as if this were the case, the stiffer PEN molecule would have a lower creep rate. A hypothesis is proposed, that creep predominantly involves the failure of secondary bonds; however this needs to be confirmed.

\section{Acknowledgments}

This study was part of a joint industrial project partly financially supported by the French CEP\&M (Comité d'Etudes Pétrolières et Marines). The authors would like to thank MM. Favry, Teissedre and Le Clerc and Mrs Piant for their technical and scientific support. They are grateful to Performance Fibers for supplying the yarns. 


\section{References}

1 - J.F. Flory, S.J. Banfield, C. Berryman (2007) Polyester Mooring Lines on Platforms and MODUs in Deep Water. Offshore Technology Conference Proceedings OTC 18768 2 - A.C. Fernandes, C.J.M. Del Vecchio, G.A.V. Castro (1998) Mechanical Properties of Polyester Mooring Cables. $8^{\text {th }}$ International Offshore and Polar Engineering Conference Proceedings, Montréal, Canada

3 - De Pellegrin I (1999) Manmade fiber ropes in Deepwater Mooring Applications. Offshore Technology Conference Proceedings OTC 10907

4 - C.J.M. Van Den Heuvel, E.A. Klop (2000) Relations between spinning, molecular structure and end-use properties of polyethylene naphthalate tyre yarns. Polymer 41:4249-4266

5 - Wu G, Li Q, Cuculo JA (2000) Fiber structure and properties of poly(ethylene-2,6naphthalate) obtained by high-speed melt spinning. Polymer 41:8139-8150

6 - E.L. Bedia, S. Murakami, T. Kitade, S. Kohjiy (2001) Structural development and mechanical properties of polyethylene naphthalate / polyethylene terephthalate blends during uniaxial drawing. Polymer 42:17:7299-7305

7 - A.R. Bunsell, J.W.S. Hearle, R.D. Hunter (1971) An apparatus for fatigue-testing of fibres. Journal of Physics E - Scientific Instruments 4:868-872

8 - Oudet, A.R. Bunsell (1987) Effects of structure on the tensile, creep and fatigue properties of polyester fibres. Journal of Material Science 22:4292-4298

9 - C.J.M. Van Den Heuvel, H.M. Heuvel, W.A. Faassen (1993) Molecular changes of PET yarns during stretching. Journal of Applied Polymer Science 49:925

10 - S. L. Phoenix (1975) Probability inter-fiber dependence and the asymptotic strength distribution of classic fiber bundles. International Journal of Engineering Science 13:287304

11 - D.C. Prevorsek, P.J. Harget, R.K. Sharma et al. (1973) Nylon 6. fibers: changes in structure between moderate and high draw ratios. Reimschussel, AC Journal of Macromolecule Science Physic 8

12 - A. Peterlin (1975) Structural model of mechanical properties and failure of crystalline polymer solids with fibrous structure. International Journal of Fracture 11:761-780

13 - J.M. Herrera Ramirez (2004) Les mécanismes de fatigue dans les fibres thermoplastiques. PhD Thesis Mines ParisTech

14 - C.J. Burgoyne, K.G.N.C. Alwis (2008) Visco-elasticity of aramid fibres. Journal of Material Science 43: 7091-7101

\section{Figures}

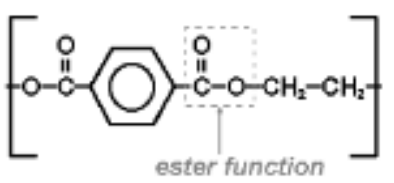

PET

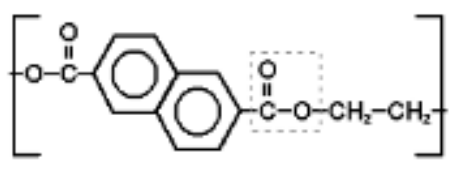

PEN

Fig. 1 Molecular structure of PET and PEN

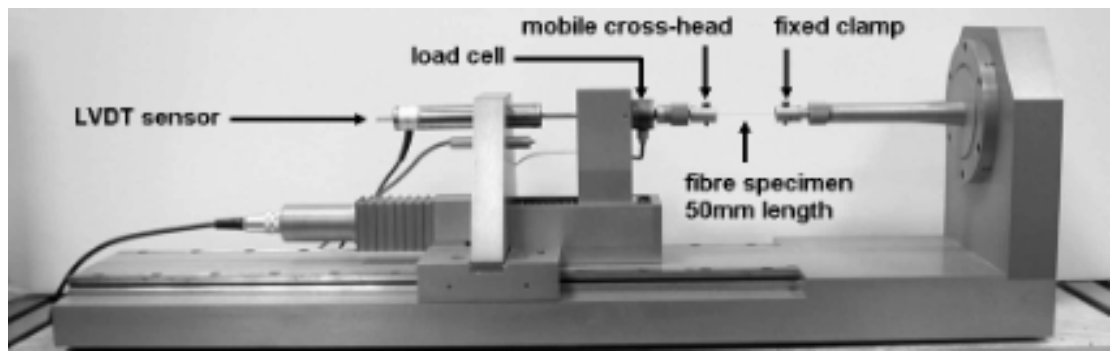

Fig. 2 Apparatus used for tensile and creep tests on fibres 

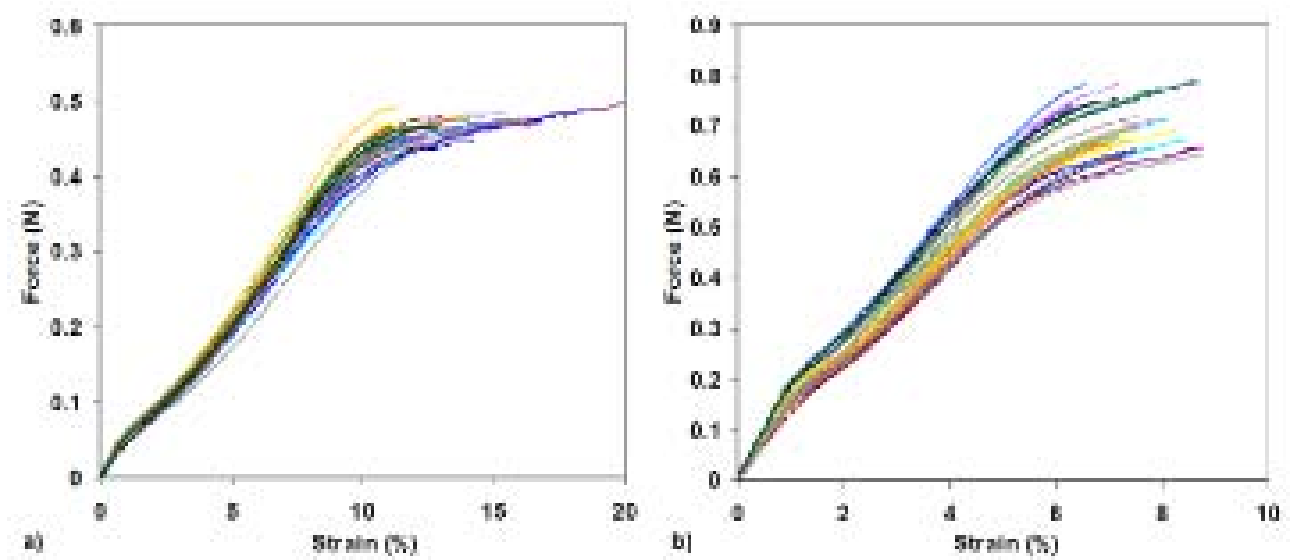

Fig. 3 Load versus strain curves from tensile tests on PET (a) and PEN (b) fibres
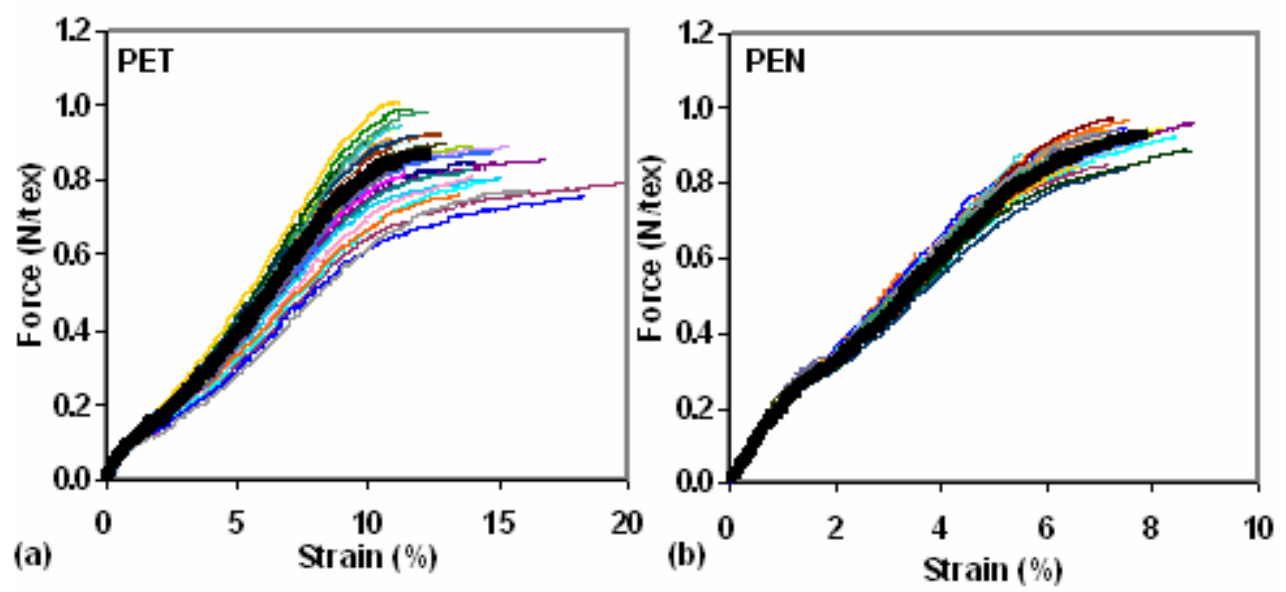

Fig. 4 Normalised Load versus strain curves from tensile tests on PET (a) and PEN (b) fibres
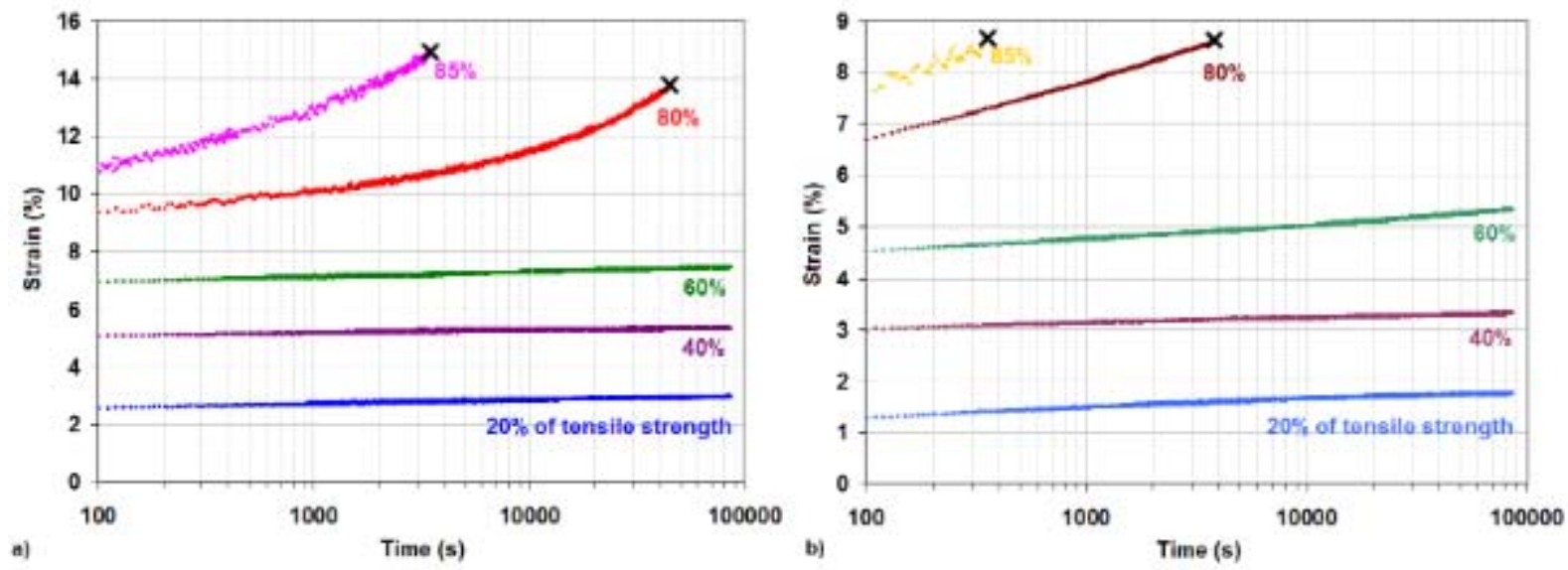

Fig. 5 Strain versus time curves from creep tests on PET (a) and PEN (b) fibres 

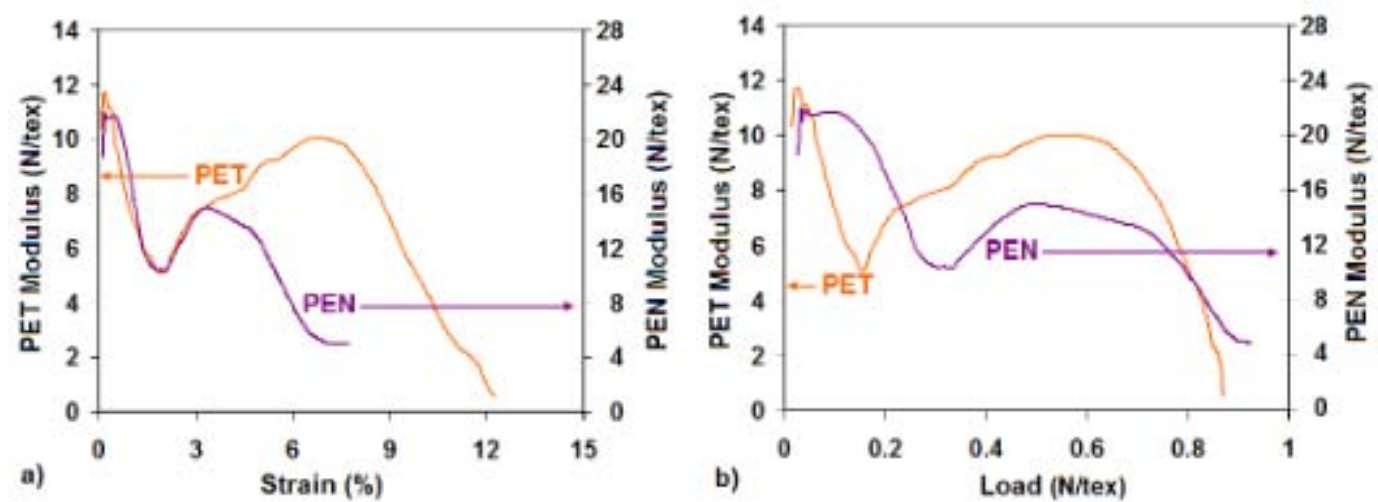

Fig. 6 Evolution of modulus with strain (a) and force (b) for an average tensile curve of PET and PEN fibre

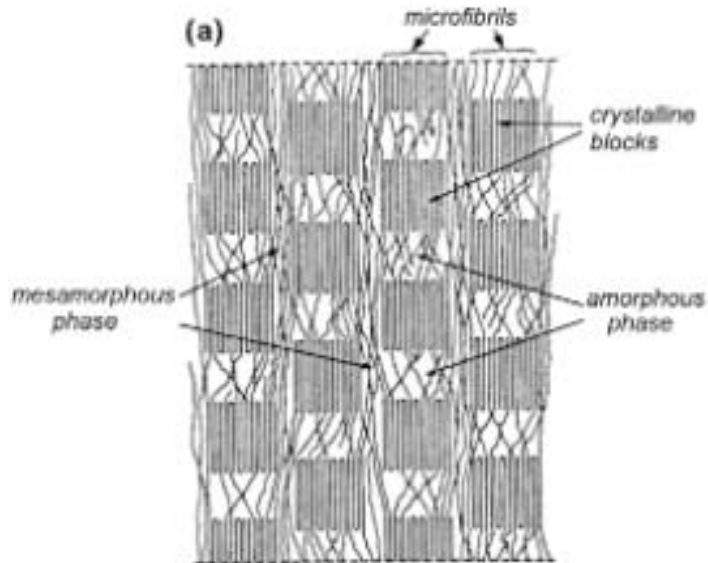

(b)

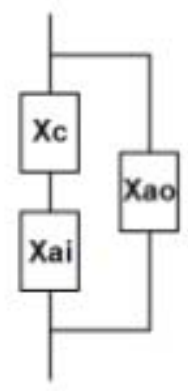

Fig. 7 (a) Microsctructural morphology of PET fibres, from Prevorsek et al. [11], and (b) corresponding schematic model $(\mathrm{Xc}=$ crystalline phase, $\mathrm{Xai}=$ amorphous isotrope phase, Xao $=$ amorphous oriented or mesamorphous phase)

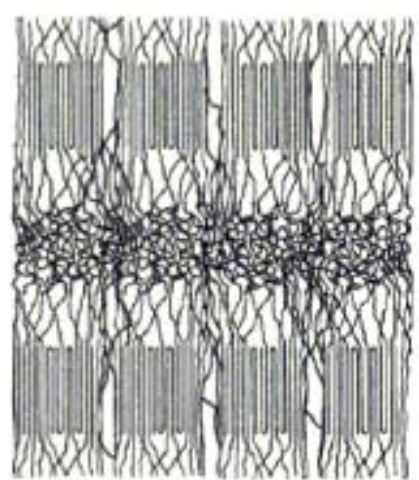

(a)

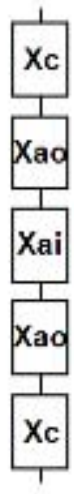

(b)

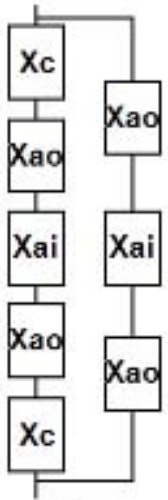

(c)

Fig. 8 (a) Microstructural morphology for PET and PEN fibres, (b) corresponding schematic model, and (c) suggested improved model for PET and PEN fibres 


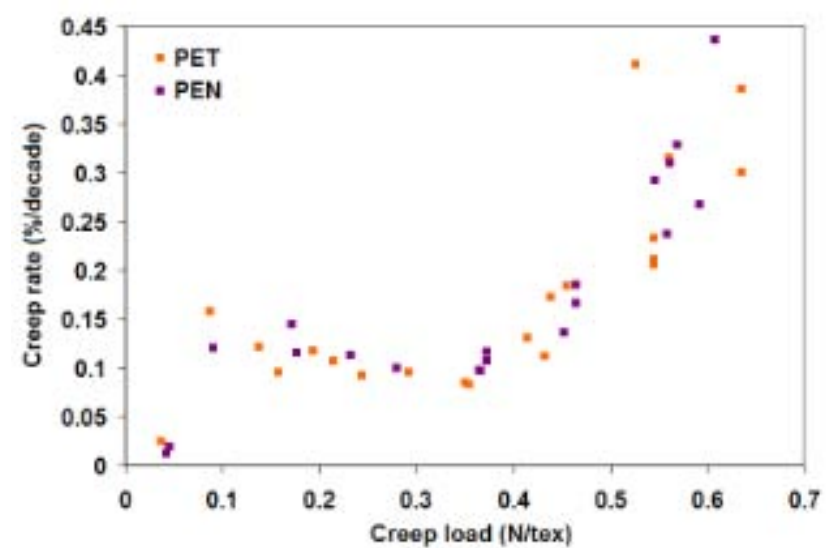

Fig. 9 Evolution of creep rate with applied load for PET and PEN fibres 